Revue internationale P.M.E.

Économie et gestion de la petite et moyenne entreprise

\title{
Une lecture entrepreneuriale de l'internationalisation des PMI sous-traitantes
}

\section{Valérie Barbat et Martine Hlady Rispal}

Volume 20, numéro 3-4, 2007

URI : https://id.erudit.org/iderudit/1008530ar

DOI : https://doi.org/10.7202/1008530ar

Aller au sommaire du numéro

Éditeur(s)

Presses de l'Université du Québec

ISSN

0776-5436 (imprimé)

1918-9699 (numérique)

Découvrir la revue

Citer cet article

Barbat, V. \& Hlady Rispal, M. (2007). Une lecture entrepreneuriale de l'internationalisation des PMI sous-traitantes. Revue internationale P.M.E., 20(3-4), 219-248. https://doi.org/10.7202/1008530ar
Résumé de l'article

Dans quelle mesure le comportement entrepreneurial de la PMI sous-traitante affecte-t-il la manière dont elle s'internationalise ? L'article se fonde sur une étude des modalités et du processus d'internationalisation des PMI sous-traitantes françaises menée dans le cadre d'un travail multiméthodes. Il montre que la subordination économique du sous-traitant au donneur d'ordres constitue un frein au développement d'une démarche entrepreneuriale susceptible d'être levée à l'international. Ainsi, la modalité d'internationalisation " autonome » traduit explicitement un comportement entrepreneurial contrairement aux autres modalités où l'internationalisation est soutenue par un ou plusieurs des acteurs du réseau d'affaires de la PMI sous-traitante. 


\title{
Une lecture entrepreneuriale de l'internationalisation des PMI sous-traitantes
}

\author{
Valérie BARBAT \\ École de management de Bordeaux \\ Martine HLADY RISPAL \\ Université Montesquieu-Bordeaux IV
}

MOTS CLÉS
PMI sous-traitantes - Processus d'internationalisation
Comportement entrepreneurial - Méthode mixte Analyse typologique - Études de cas

\begin{abstract}
LES AUTEURES
VALÉRIE BARBAT détient un doctorat en sciences de gestion. Elle est professeure à BEM (École de management de Bordeaux), responsable du Pôle Marketing, membre du CEREBEM et membre invitée de l'équipe Entrepreneuriat de l'IRGO (Université Montesquieu-Bordeaux IV). Ses travaux se concentrent sur l'internationalisation des PMI sous-traitantes. En outre, elle s'intéresse aux parcs industriels de fournisseurs. Adresse: BEM, 680, cours de la Libération, 33405 Talence Cédex, France. Courriel: <valerie.barbat@ bordeaux-bs.edu>.

Martine HLAdY RISPal détient un doctorat en sciences de gestion. Elle est maître de conférences à l'Université Montesquieu-Bordeaux IV et membre des équipes Entrepreneuriat et Marketing du Laboratoire IRGO (Institut de recherche en gestion des organisations). En plus de s'intéresser fortement aux méthodes qualitatives, elle mène des travaux sur les stratégies relationnelles entre responsables de petites entités. Adresse: PUSG, 35, avenue Abadie, 33072 Bordeaux, France. Courriel: <martine.hlady@u-bordeaux4.fr>.
\end{abstract}

\section{RÉSUMÉ}

Dans quelle mesure le comportement entrepreneurial de la PMI sous-traitante affecte-t-il la manière dont elle s'internationalise? L'article se fonde sur une étude des modalités et du processus d'internationalisation des PMI sous-traitantes françaises menée dans le cadre d'un travail multiméthodes. II montre que la subordination économique du sous-traitant au donneur d'ordres constitue un frein au développement d'une démarche entrepreneuriale susceptible d'être levée à l'international. Ainsi, la modalité d'internationalisation «autonome » traduit explicitement un comportement entrepreneurial contrairement aux autres modalités où l'internationalisation est soutenue par un ou plusieurs des acteurs du réseau d'affaires de la PMI sous-traitante. 


\begin{abstract}
To what extent does entrepreneurial behaviour of a sub-contracting SME affect the way in which it internationalizes itself? Our article is based on a study of the methods and internationalization process of sub-contracting French SME carried out using multi-methods research. It shows that the economic subordination of the subcontractor to the principal constitutes a structural obstacle to the development of an entrepreneurial approach likely to disappear through internationalization. Thus the "autonomous" internationalization mode explicitly translates an entrepreneurial behaviour contrary to the other modes where internationalization is supported by one or more actors belonging to the network of the sub-contracting SME.
\end{abstract}

\title{
RESUMEN
}

¿En qué medida el comportamiento empresarial de la PyME subcontratista afecta la manera en que se internacionaliza? El artículo presenta el estudio de las modalidades y del proceso de internacionalización de PyME subcontratistas francesas movilizando diferentes métodos de investigación. Demuestra que la subordinación económica del subcontratista al dador constituye un freno al desarrollo de un comportamiento empresarial que puede desaparecer cuando se internacionaliza. Entonces la modalidad de internacionalización "autónoma" traduce explícitamente un comportamiento empresarial contrariamente a las otras modalidades donde la internacionalización está respaldada por unos o más de los protagonistas de la red de negocios de la PyME subcontratista.

\section{ZUSAMMENFASSUNG}

In welchem Masse beeinflusst das unternehmerische Verhalten von KMI Zulieferbetrieben die Art und Weise ihres Internationalisierungsprozesses? Der Artikel basiert auf einer Studie über die Modalitäten und Prozesse der Internationalisierung von französischen KMI Zulieferbetrieben, die im Rahmen einer methodisch vielfältigen Arbeit durchgeführt wurde. Er zeigt auf, dass die wirtschaftliche Unterordnung des Zulieferers ein Hemmnis darstellt, dass die unternehmerischen Bestrebungen im Hinblick auf die Internationalisierung bremst. Folglich ist die „autonome“ Internationalisierungsform eine Konsequenz aus unternehmerischem Verhalten. Dies in Abgrenzung zu Internationalisierungsformen, bei denen der Prozess massgeblich durch Geschäftspartner gestützt ist.

\section{Introduction}

Longtemps, la recherche de débouchés extérieurs au marché domestique ne constituait qu'une préoccupation marginale des sous-traitants français (Altersohn, 1992). C'est au cours des années 1990 que l'affirmation de cette vocation internationale a connu une évolution sensible tant à l'échelle nationale qu'européenne (SESSI, 2003). Traditionnellement peu exportatrices, les PMI dont l'activité de sous-traitance représente plus de $80 \%$ de leur 
chiffre d'affaires affichent néanmoins, depuis 1998, un taux d'exportation moyen aux alentours de $20 \%$ (L'Usine Nouvelle, 2003). Cet engagement récent sur les marchés extérieurs tend à bouleverser le paysage de la soustraitance industrielle française en favorisant la sous-traitance de spécialité au détriment de la sous-traitance de capacité ${ }^{1}$. Par ailleurs, l'engagement ou non des sous-traitants à l'international a des conséquences sur l'emploi et le dynamisme économique tant au plan national que local. Le développement international de la sous-traitance industrielle revêt d'autant plus d'importance que la sous-traitance confiée représente en France environ $12 \%$ du chiffre d'affaires annuel de l'ensemble de l'industrie manufacturière. En 2003, le SESSI observait que plus de 7200 entreprises de plus de 20 salariés appartenant aux secteurs spécialisés dans la sous-traitance industrielle avaient réalisé 72 milliards d'euros de facturation ${ }^{2}$. Parmi ces secteurs, ceux des métaux et des plastiques concentraient près de la moitié de l'activité de la sous-traitance industrielle avec 32 milliards d'euros. Ces sous-traitants sont à $95 \%$ des entreprises de moins de 500 salariés.

En dépit de son importance économique, ce phénomène souffre d'un déficit de traitement sur un plan académique. Or, le sujet est intéressant à plus d'un titre. En raison des caractéristiques majeures des entreprises étudiées leur taille et leur qualité de sous-traitant -, deux acteurs principaux sont susceptibles d'orienter leur internationalisation: le dirigeant-propriétaire et le client donneur d'ordres. Ce constat invite à s'interroger sur le rôle de chacun de ces acteurs dans la définition des modalités d'accès à l'exportation de leur entreprise. Il conduit ainsi à examiner l'existence d'un lien potentiel entre les modalités effectivement mises en œuvre et le développement, par les PMI sous-traitantes, d'un comportement entrepreneurial défini par Burgelman (1983) comme la capacité qu'ont les dirigeants à reformuler sans

1. Pour l'AFNOR (Association française de normalisation), «la sous-traitance est dite de capacité dès lors que l'entreprise donneuse d'ordres, équipée elle-même pour exécuter un produit, a recours à une autre entreprise soit occasionnellement, en raison d'une pointe momentanée dans sa production ou d'un incident technique, soit de façon plus ou moins habituelle parce que, désireuse de conserver une capacité propre dans une fabrication déterminée, elle entend utiliser des capacités de production disponibles à l'extérieur;» tandis que «la sous-traitance est dite de spécialité dès lors que l'entreprise donneuse d'ordres décide de faire appel à un spécialiste disposant des équipements et de la compétence adaptés à ses besoins, parce qu'elle ne peut pas ou ne souhaite pas, pour des raisons relevant de sa stratégie propre, notamment des considérations d'ordre économique, se doter des moyens nécessaires au lancement ou au développement d'une fabrication, ou encore parce qu'elle estime ses installations insuffisantes ou insuffisamment compétitives pour cette fabrication» (SESSI, 2000, p. 3).

2. La sous-traitance industrielle française représente $18,5 \%$ de la sous-traitance européenne et se situe au deuxième rang derrière l'Allemagne. 
cesse leur environnement. Notre travail se situe également dans la mouvance de travaux de recherche épars tels ceux d'Andersson (2000) sur la dimension entrepreneuriale dans l'internationalisation des PME ou ceux consacrés à l'entrepreneuriat international (McDougall et Oviatt, 2000 et 2005) qui définissent le comportement entrepreneurial transfrontalier comme celui d'entreprises qui recherchent et exploitent des opportunités à l'étranger afin de créer de nouveaux produits et services. La problématique de notre recherche peut dès lors être formulée sous un mode interrogatif: dans quelle mesure le comportement entrepreneurial de la PMI sous-traitante affecte-t-il la manière dont elle s'internationalise?

L'étude empirique multiméthodes combinant une enquête par questionnaires auprès de 103 PMI sous-traitantes françaises et huit études de cas exemplaires extraites de l'analyse typologique réalisée préalablement fait émerger quatre modalités d'internationalisation susceptibles d'être regroupées en deux grandes logiques d'internationalisation génériques: la logique «entrepreneuriale» et la logique «induite réactive».

Notre objectif est double. Il convient, dans un premier temps, d'établir dans quelle mesure l'internationalisation des PMI sous-traitantes s'inscrit potentiellement ou clairement dans une logique entrepreneuriale. De façon plus émergente, il s'agit d'explorer la nature de cette logique. Notre article invite le lecteur à s'interroger sur la nature entrepreneuriale de la PMI soustraitante dans un contexte international. À partir d'une étude empirique combinant une enquête par questionnaires et des études de cas, deux logiques d'internationalisation émergent dont une traduit le comportement entrepreneurial de la PMI sous-traitante. La diversité des formes entrepreneuriales relevées est enfin explorée.

\section{La sous-traitance à l'international, un contexte propice à l'entrepreneuriat?}

En raison de la nature particulière de la relation de sous-traitance industrielle, la stratégie du sous-traitant est influencée à des degrés divers, parfois très élevés, par ses donneurs d'ordres. En conséquence, la PMI sous-traitante n'offre pas, de prime abord, un contexte favorable à l'entrepreneuriat (1.1). Cette affirmation peut-elle être réformée lorsque le sous-traitant est engagé à l'international? Les modèles d'internationalisation font partiellement référence à l'esprit d'entreprise du dirigeant de la PME/PMI (1.2). 


\subsection{La relation de sous-traitance, un frein au développement d'un comportement entrepreneurial}

La sous-traitance industrielle est une réalité économique complexe difficile à circonscrire. Son évolution depuis les années 1980 a conduit à la multiplication des formes de sous-traitance et à la porosité des frontières entre soustraitance et fourniture de produits standard. Néanmoins, une composante commune discrimine la sous-traitance des autres formes de coopération interentreprises: la subordination économique du sous-traitant au donneur d'ordres (Barbat, 2004). Cette subordination naît de l'existence d'un cahier des charges - technique ${ }^{3}$ ou fonctionnel ${ }^{4}$ - préétabli par le donneur d'ordres et auquel le sous-traitant doit se conformer. L'autonomie technique du soustraitant peut varier d'une situation à l'autre, il peut ou non intervenir lors de la phase de conception et l'ingérence du donneur d'ordres peut être plus ou moins forte, le client assume toujours en dernier ressort la responsabilité de la conception du produit final et les possibilités de sa commercialisation.

Plus qu'une entité physique dotée d'utilités fonctionnelles, elle inclut la capacité du sous-traitant à livrer en temps et lieu une opération technique parfaitement adaptée aux exigences du client, mais aussi sa capacité à flexibiliser l'offre à des demandes futures, à suggérer des innovations (Baudry, 1999). Dans ces conditions, le sous-traitant peut difficilement redéployer ses actifs sans délai et sans coût vers un autre donneur d'ordres, contrairement à un fournisseur de produits standard.

Les donneurs d'ordres sont souvent qualifiés d'entrepreneurs, notamment lorsqu'ils font appel à des sous-traitants à l'international, de preneurs de risques ou de profils innovants, traits caractéristiques des entrepreneurs. En revanche, les PMI sous-traitantes, en raison du lien de subordination, apparaissent comme étant l'antithèse d'entreprises entrepreneuriales. Elles opèrent dans le cadre d'une organisation centrée sur l'économie de coûts de production sous les directives techniques ou fonctionnelles d'un donneur d'ordres: «Du seul fait qu'il est subordonné à un client appelé donneur d'ordres, le sous-traitant peut difficilement ne pas être considéré comme la victime d'un diminutio capitis, d'une fatalité qui le condamne à être un entrepreneur d'une espèce inférieure»(Altersohn, 1992, p. 16). Avec le

3. «Le cahier des charges technique est l'expression de la volonté du donneur d'ordres sur l'objet qu'il assigne au contrat et qui est fonction du résultat industriel qu'il recherche.» (Commission européenne, 1992, p. 7.)

4. «Le cahier des charges dit fonctionnel comporte des spécifications d'objectifs ou de performances mais rend l'exécutant responsable du choix des caractéristiques du produit sous réserve du respect de certaines contraintes liées à des conditions d'utilisation et de fonctionnement.» (Altersohn, 1992, p. 27.) 
développement du partenariat dans les années 1980, les mécanismes de coordination de la relation de sous-traitance alors exclusivement autoritaires ${ }^{5}$ deviennent peu à peu plus incitatifs ${ }^{6}$. Néanmoins, les décisions et objectifs d'une PMI sous-traitante, son développement national ou international mais aussi sa survie ${ }^{7}$, ne sont jamais complètement isolés de la stratégie de ses principaux donneurs d'ordres, rarement très nombreux. Ces derniers, dès qu'ils le peuvent, tendent à s'ingérer dans la gestion de leurs sous-traitants : en contrôlant leurs outils de production (sous-traitance à façon), en évaluant leur système qualité, en participant à la gestion de leurs ressources humaines (Laval, 1994), en initiant leur regroupement (Barbat, 1996), en servant de rampe d'accès à toutes sortes de ressources externes pour compenser leur manque de ressources de base par le portage ou le soutien au déploiement vers d'autres clients pour compenser une baisse d'activité sous-traitée (Barbat, 2004).

Pour toutes ces raisons, le rôle du dirigeant de la PMI sous-traitante s'apparenterait plus au rôle traditionnellement attribué au gestionnaire, au manager et à l'organisateur qu'à l'entrepreneur (Julien et Marchesnay, 1989; Stewart et al.,1999). La qualité de sous-traitant constitue, dès lors, un frein au comportement entrepreneurial de ces entreprises. En définitive, la relation de

5. Parmi les dispositifs autoritaires utilisés par les donneurs d'ordres, la mise en concurrence est l'un des plus célèbres. Il s'agit d'une politique d'achat qui consiste à stimuler la rivalité entre les fournisseurs et à reporter continuellement les commandes du donneur d'ordres vers la source d'approvisionnement la plus compétitive (Fenneteau, 1990). La non-efficience du sous-traitant engendrée par ce dispositif (la mise en concurrence empêchant le sous-traitant de planifier ses investissements, interdisant tout effet d'expérience et limitant ses innovations) conduit les donneurs d'ordres à recourir à l'approvisionnement à partir d'une source unique (single sourcing).

6. Les mécanismes incitatifs visent à l'allongement de la durée de l'engagement du sous-traitant par le recours à un contrat, de type contrat-cadre (Baudry, 1991), de plus long terme, renouvelable. Ils représentent pour le sous-traitant l'espérance de gains futurs sur une durée relativement importante.

7. L'histoire des PME sous-traitantes du Choletais (de 1945 à 2004) proposée par Courault (2005) est, à ce titre, révélatrice. Les grandes entreprises (GE) du secteur de l'habillement de cette région «avaient créé au cours des années 1960 de nombreuses unités sous-traitantes, qui leur appartenaient souvent en propre, mais étaient aussi indépendantes. Elles assuraient la plus grosse partie des commandes de ces unités, qui étaient soit des sous-traitants exclusifs, soit des partenaires privilégiés. Lorsque les GE se sont restructurées, la première chose qu'ont faite les repreneurs ou les nouveaux dirigeants a consisté à mettre un terme à ces relations privilégiées. Les PME sous-traitantes furent ainsi forcées de se réorganiser sous peine de disparaître [...] Beaucoup de ces PME sous-traitantes ne purent faire face» (p. 27). 
sous-traitance industrielle entre un donneur et un preneur d'ordres de taille restreinte renvoie souvent à la perception d'une relation entre un innovateur/ entrepreneur et un réalisateur/manager.

\subsection{L'internationalisation, vecteur potentiel d'un comportement entrepreneurial}

Il est possible de déplacer le regard de la relation donneur/preneur d'ordres en retenant la perspective de la PMI sous-traitante sous l'angle de la prise de risque, de la coopération, de l'exploitation d'un réseau d'affaires. Cette perspective est permise grâce à l'internationalisation de la PMI sous-traitante.

Plusieurs acteurs sont susceptibles de jouer un rôle phare dans le développement d'un savoir-faire à l'international. Tout d'abord, le dirigeant, au centre de l'organisation de taille restreinte (Julien et Marchesnay, 1989). Ses perceptions, attitudes et attentes à l'égard de l'international mais aussi des caractéristiques telles que l'origine culturelle ou la formation, le fait d'avoir vécu à l'étranger, d'avoir une expérience professionnelle préalable à l'international (Bloodgood, Sapienza et Almeida, 1996; Shrader, Oviatt et McDougall, 2000), influencent sensiblement le processus d'internationalisation de la PMI. En raison de la nature de la relation de sous-traitance, les donneurs d'ordres interviennent également en tant que force initiatrice ou soutien à l'internationalisation de la PMI sous-traitante (Andersen, Blenker et Christensen, 1995 ; Holmlund et Kock, 1998). On observe enfin le concours des organismes institutionnels et privés d'accompagnement à l'exportation (Nummela, 1997) ainsi que celui des entreprises de taille équivalente dans le cadre d'accords de coopération français ou européens (Rispal, 1993; Hovi, 1994). Le dirigeant et les réseaux d'affaires sont, par conséquent, au cœur de la stratégie exportatrice des PMI sous-traitantes. Dans ce contexte, le développement international de la PMI sous-traitante doit être appréhendé comme un processus d'acquisition de ressources, en interne et en externe, à même de créer le potentiel et les conditions nécessaires à la satisfaction d'un donneur d'ordres étranger. Notre étude privilégie alors, à l'instar de récents écrits (Coviello et McAuley, 1999; Johanson et Vahlne, 2003; Dalgic, Li et Li, 2004), la complémentarité des approches «béhavioriste» (Johanson et Wiedersheim-Paul, 1975; Johanson et Vahlne, 1977; Bilkey et Tesar, 1977) et par les «réseaux» (Johanson et Mattsson, 1988; Bell,1995; Coviello et Munro, 1997) de l'internationalisation. L'approche béhavioriste constitue une grille d'analyse pour décrire les séquences du processus d'internationalisation tout en privilégiant l'acquisition interne des ressources nécessaires au développement international de la PMI. L'intérêt de l'approche réseau réside dans le fait d'intégrer à l'analyse des modalités du développement international de l'entreprise, l'influence des acteurs de son réseau et, plus particulièrement, des donneurs d'ordres dans le cas qui nous intéresse. 
Parce qu'il présente des caractéristiques d'innovation, de proactivité, de prise de risque, de capacité à créer et à saisir des opportunités au sein d'un réseau, le processus d'internationalisation est potentiellement sous-tendu par une ou plusieurs des quatre dimensions entrepreneuriales énoncées par Verstraete et Fayolle (2005): la création d'organisations, la recherche d'opportunités, la création de valeur ou encore l'innovation.

La première dimension montre que l'organisation entrepreneuriale est en premier lieu une entreprise qui crée de nouvelles organisations (Gartner, 1993; Thornton, 1999) au sein desquelles l'intensité du changement pour l'individu et l'importance de la création de valeur se situent à des niveaux élevés. Le concept d'impulsion développé par Verstraete suggère une dynamique de création et de développement, l'action d'entreprendre aussi bien que son résultat et dépasse la notion de simple création d'entités nouvelles. Bien que la littérature sur l'internationalisation des PME/PMI retienne l'exportation comme modalité principale de l'internationalisation de ces entreprises, il n'en demeure pas moins que certaines d'entre elles, pour une part non négligeable, mettent en œuvre des stratégies d'internationalisation aboutissant à la création de filiales à l'étranger (McDougall, Shane et Oviatt, 1994). Concernant la population qui nous intéresse ici, à savoir les PMI sous-traitantes, une enquête réalisée par L'Usine Nouvelle en 1996 auprès de 243 entreprises montre que $13 \%$ de ces entreprises possèdent des implantations industrielles hors de nos frontières.

Pour Shane et Venkataraman (2000), la création de nouvelles organisations n'est pas une condition de l'entrepreneuriat. Ces auteurs, à l'instar de Stevenson et Jarillo (1990) et Bygrave et Hofer (1991), privilégient la dimension de découverte, d'évaluation et d'exploitation d'opportunités pour caractériser l'organisation entrepreneuriale. L'acteur qui découvre et exploite une occasion d'affaires est essentiel à la dynamique d'internationalisation (McDougall et Oviatt, 2005). Wolff et Pett (2000) montrent que la décision d'exporter dépend en grande partie de l'existence d'opportunités. Toutefois, une occasion d'affaires ne vaut que si l'entreprise est en mesure de la saisir (Reid, 1981; Flechter et Vyakarnam, 1999). Compte tenu du rôle prédominant joué par le dirigeant dans la gestion de la PME, de nombreux travaux s'intéressent aux caractéristiques personnelles et traits psychologiques du dirigeant pour expliquer la décision d'exporter (Wiedersheim-Paul, Olson et Welch, 1978; Reid, 1981; Dichtl et al., 1984; Lloyd-Reason et Mughan, 2002), l'intention d'exporter (Jaffe et Pasternak, 1994) ou, plus largement, le développement international de l'entreprise (Harveston, Kedia et Davis, 2002). D'autres études indiquent que la capacité du dirigeant de la PME à s'insérer dans des réseaux d'affaires et/ou personnel d'envergure internationale est source d'opportunités pour la PME (McDougall, Shane et Oviatt, 1994; Coviello et Munro, 1995; Barbat, 2004).

Revue internationale P.M.E., vol. 20, nos 3-4, 2007 
Au-delà de l'opportunité détectée dont il faut se saisir, l'exportation et, à fortiori, l'internationalisation posent le problème de la capacité de la $\mathrm{PME}$ à développer ou à créer les ressources organisationnelles permettant de gérer les défis qu'elle engendre (Ageron, 2001). Dans cette perspective, l'internationalisation de la PME peut aboutir à une recomposition de sa chaîne de valeur. Le dirigeant, même s'il n'est plus tout seul, continue de jouer un rôle important dans cette activation de la création de valeur au sein de son entreprise.

La dimension de «création de valeur», développée notamment par Ronsdadt (1984) et Bruyat (1993) pour caractériser l'organisation entrepreneuriale au plus près de la réalité des petites entités, est, par conséquent, omniprésente dans le processus d'internationalisation. Les auteurs insistent sur le rôle fondamental de l'entrepreneur, individu acteur principal et décisionnaire, créateur de richesse et de valeur pour l'entité. Sa responsabilité est d'infuser la valeur en sécurisant et en allouant les compétences et les ressources nécessaires au développement de produits et services.

Enfin, Verstraete et Fayolle (2005) constatent que l'innovation est fréquemment associée à l'entrepreneuriat, notamment pour les auteurs centrés sur les entreprises de haute technologie ou la valorisation économique des travaux de recherche. Or, les PME les plus exportatrices sont aussi celles qui innovent le plus et qui font le plus de recherche-développement (Wolff et Pett, 2000).

Les définitions les plus récentes de l'entrepreneuriat international des PME retiennent ainsi la dimension de découverte, de construction et d'exploitation d'opportunités transfrontalières dans le but de créer des produits et services radicalement nouveaux (McDougall et Oviatt, 2000 et 2005).

\section{La méthodologie de l'étude empirique}

Le champ de notre investigation empirique est celui des PMI (moins de 500 salariés) sous-traitantes (dont l'activité de sous-traitance représente plus de $80 \%$ du chiffre d'affaires) françaises, juridiquement autonomes qui établissent régulièrement des relations de sous-traitance directes avec des donneurs d'ordres localisés à l'étranger. Afin d'observer des entreprises significativement exportatrices, nous avons retenu celles exportant au moins $5 \%$ de leur chiffre d'affaires. L'orientation de notre objet de recherche de nature exploratoire nous conduit à adopter une stratégie de recherche mixte combinant une enquête par questionnaires et des entretiens en profondeur auprès de dirigeants de PMI sous-traitantes (voir tableau 1). 


\section{TABLEAU 1}

\section{Démarche méthodologique de l'étude empirique}

\begin{tabular}{|c|c|c|}
\hline $\begin{array}{c}\text { Entretiens exploratoires } \\
\text { MÉTHODE QUALITATIVE } \\
\text { - Se familiariser avec le } \\
\text { phénomène étudié. } \\
\text { - Préparer } \\
\text { l'enquête terrain. } \\
\end{array}$ & \begin{tabular}{|c|} 
Enquête \\
par questionnaires \\
MÉTHODE QUANTITATIVE \\
- Identifier et décrire les \\
modalités d'exportation \\
des PMI sous-traitantes. \\
\end{tabular} & $\begin{array}{l}\text { Entretiens en profondeur } \\
\text { MÉTHODE QUALITATIVE } \\
\text { - Comprendre } \\
\text { la dynamique de } \\
\text { construction du processus } \\
\text { d'internationalisation. } \\
\end{array}$ \\
\hline $\begin{array}{l}\text { Recueil des données } \\
\text { Entretiens non directifs } \\
\text { et semi-directifs }+ \\
\text { rapports d'experts. }\end{array}$ & $\begin{array}{l}\text { Recueil des données } \\
\text { Questionnaires adminis- } \\
\text { trés par voie postale. }\end{array}$ & $\begin{array}{l}\text { Recueil des données } \\
\text { Entretiens semi-directifs. }\end{array}$ \\
\hline $\begin{array}{l}\text { Échantillon } \\
4 \text { experts }+11 \text { dirigeants } \\
\text { de PMI sous-traitantes. }\end{array}$ & \begin{tabular}{|l|} 
Échantillon \\
103 PMI sous-traitantes \\
engagées à l'exportation.
\end{tabular} & \begin{tabular}{|l} 
Échantillon \\
8 PMI sous-traitantes \\
exemplaires des classes \\
formées par l'analyse \\
typologique.
\end{tabular} \\
\hline $\begin{array}{l}\text { Modes d'analyse } \\
\text { Fiches de synthèse, } \\
\text { d'entretiens } \\
\text { et de documents. }\end{array}$ & \begin{tabular}{|l} 
Modes d'analyse \\
Analyse typologique, \\
analyse discriminante, \\
analyse de variance, \\
test du Chi-deux (SPSS).
\end{tabular} & $\begin{array}{l}\text { Modes d'analyse } \\
\text { Codage, résumé du } \\
\text { contexte, matrices } \\
\text { et graphiques intra- } \\
\text { et intersites. } \\
\end{array}$ \\
\hline
\end{tabular}

\subsection{Les objectifs recherchés}

Étant donné l'intérêt récent des chercheurs pour l'internationalisation des PMI sous-traitantes, une étude exploratoire s'est rapidement avérée indispensable afin de nous familiariser avec le phénomène étudié et de préparer notre investigation terrain en termes d'élaboration des modalités de l'enquête et du questionnaire. L'enquête par questionnaires avait pour objet de repérer les modalités d'exportation auprès d'un échantillon d'entreprises à un moment déterminé dans le temps et, par conséquent, d'apprécier précisément l'étendue du rôle d'initiateur et/ou d'accompagnateur des donneurs d'ordres au cours du processus d'internationalisation des PMI sous-traitantes. Elle offrait également un premier niveau de décryptage de ces modalités à l'aide principalement des caractéristiques internes de l'entreprise et de son dirigeant.

Toutefois, en raison de sa nature éminemment synchronique, cette méthode d'analyse nous empêchait d'introduire une dimension temporelle dynamique dans l'étude du processus d'internationalisation. Pour remédier à cet inconvénient, nous avons choisi de compléter notre enquête par questionnaires par une recherche qualitative sur un petit nombre de cas exem- 
plaires déterminés statistiquement parmi les différentes configurations de modalités d'exportation relevées. Les entretiens semi-directifs ont permis de rendre compte de l'évolution de la PMI sous-traitante par rapport au fait international et ainsi de mener une étude d'ordre processuel. En outre, elle nous offre la possibilité de mieux comprendre le rôle joué par les acteurs du réseau de la PMI sous-traitante intervenus dans son processus d'internationalisation ainsi que la nature des liens qui existent entre le sous-traitant et les acteurs de son réseau à l'origine d'un comportement potentiellement entrepreneurial.

\subsection{Les stratégies d'analyse}

À partir des résultats de l'enquête par questionnaires, une analyse typologique a été réalisée, utilisant successivement deux algorithmes de classification : la méthode de classification hiérarchique de Ward puis la méthode de classification non hiérarchique des nuées dynamiques. Ce procédé a permis de retenir une classification en quatre classes, soit les plus homogènes, et d'apprécier sa fiabilité (le taux de reclassement des individus d'une classification à l'autre atteignant 78,6 \% ). La description des groupes, à partir d'une analyse discriminante, a permis d'extraire la signification pratique des groupes. En raison de la succession des méthodes opérée au sein de la stratégie de recherche, les résultats de l'étude extensive orientent la définition de notre échantillon qualitatif. Huit PMI sous-traitantes exemplaires des quatre classes formées par l'analyse typologique ont ainsi été retenues et ont fait l'objet d'entretiens en profondeur auprès de leur dirigeant d'une durée comprise entre une heure quinze et une heure quarante-cinq (voir tableau 2).

Trois stratégies d'analyse qualitative ont été privilégiées: la stratégie narrative, la stratégie graphique qui s'appuie sur des formes matricielles et graphiques (Huberman et Miles, 2003) et une analyse thématique en trois temps. Les données recueillies ont été comparées dans un premier temps à une batterie de critères relatifs aux stimuli liés à l'internationalisation ( $c f$. tableau 4). Chaque cas a ainsi été positionné et décrit comme exemplaire d'un des quatre modes d'internationalisation relevés lors de la phase quantitative de la recherche. Les cas ont ensuite été comparés aux caractéristiques nées de l'examen de la littérature en entrepreneuriat. Ce deuxième temps a permis de renforcer la dissociation entre cas induits réactifs et cas entrepreneuriaux, puis de spécifier les traits partagés par les trois cas entrepreneuriaux (cf. § 4.1). Une analyse plus inductive des cas entrepreneuriaux a permis dans un dernier temps l'identification de quatre thèmes ou catégories développés dans la section intitulée «les traits différenciateurs» (cf. § 4.2). 


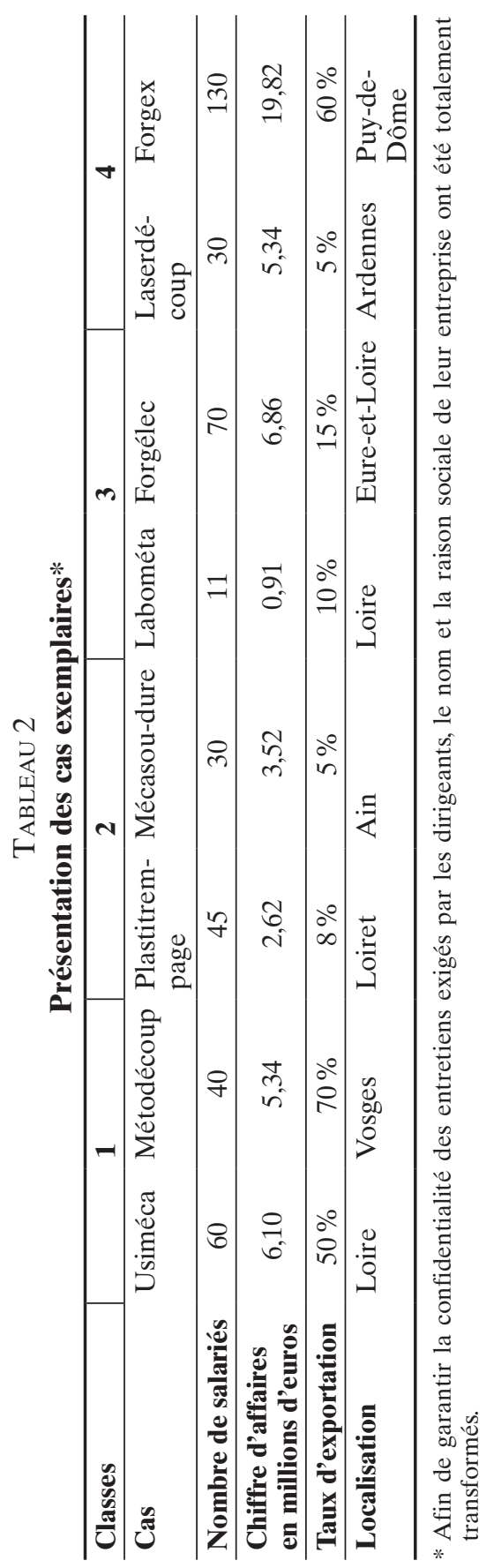

Revue internationale P.M.E., vol. 20, nos 3-4, 2007 


\section{Modalités et logiques d'internationalisation}

L'enquête par questionnaire aboutit à l'identification de quatre modalités d'internationalisation entendues comme modes d'accès à l'exportation (3.1). L'approfondissement des caractéristiques de ces modalités, grâce à l'étude de huit cas exemplaires, débouche sur l'émergence de deux grandes logiques d'internationalisation: induite réactive et entrepreneuriale ${ }^{8}$ (3.2).

\subsection{Quatre modalités d'internationalisation}

L'étude exploratoire (tableau 1) montre que les PMI sous-traitantes retiennent presque exclusivement l'exportation comme modalité principale d'internationalisation. Par ailleurs, lorsqu'elles s'internationalisent, elles sollicitent surtout le réseau de leurs donneurs d'ordres et/ou institutionnel, ce qui les amène à recourir à différents modes d'accès à l'exportation. L'analyse typologique est alors réalisée à partir de l'attribut suivant: la fréquence d'intervention des acteurs externes dans le processus d'internationalisation de la PMI soustraitante. La liste de six acteurs externes privés et institutionnels a pu être établie grâce à l'étude exploratoire : les donneurs d'ordres, les sous-traitants, les syndicats professionnels, les bourses de sous-traitance, les chambres de commerce et d'industrie et les conseils régionaux/généraux. Cette liste ne pouvant être par nature exhaustive, l'item «Autre(s): à préciser» a été ajouté.

Quatre classes de modalités d'internationalisation surviennent et se distinguent selon la nature et l'intensité du soutien du réseau d'affaires dont ont bénéficié les PMI sous-traitantes au cours de leur internationalisation (tableau 3):

1) Les moins soutenus ( $23 \%$ ) - soit 24 entreprises dont 10 n'ont bénéficié d'aucun soutien au cours de leur processus d'internationalisation et 14 faiblement soutenues plutôt par des agents commerciaux et des clients domestiques que par tout autre acteur.

2) Les suiveurs de donneurs d'ordres domestiques (35\%), qui s'internationalisent principalement dans le sillage de leurs clients, surtout domestiques. Les acteurs institutionnels interviennent nettement plus rarement.

8. Notre classification s'apparente à celle proposée par Burgelman (1983). L'auteur se situe dans le courant de l'entrepreneuriat organisationnel d'entreprises dotées d'activités diversifiées à l'international. Il explore deux types de comportements génériques: induit ou autonome. Un comportement stratégique induit respecte la planification des objectifs fixés à l'avance en termes de portefeuille d'activités et d'allocation de ressources et s'intègre dans un contexte structurel préétabli. Seule une innovation de nature incrémentale est envisageable. Un comportement stratégique autonome permet l'identification de nouveaux segments de marché, partant, la reformulation de l'environnement de l'entreprise. Une innovation radicale est rendue possible.

Revue internationale P.M.E., vol. 20, nºs 3-4, 2007 


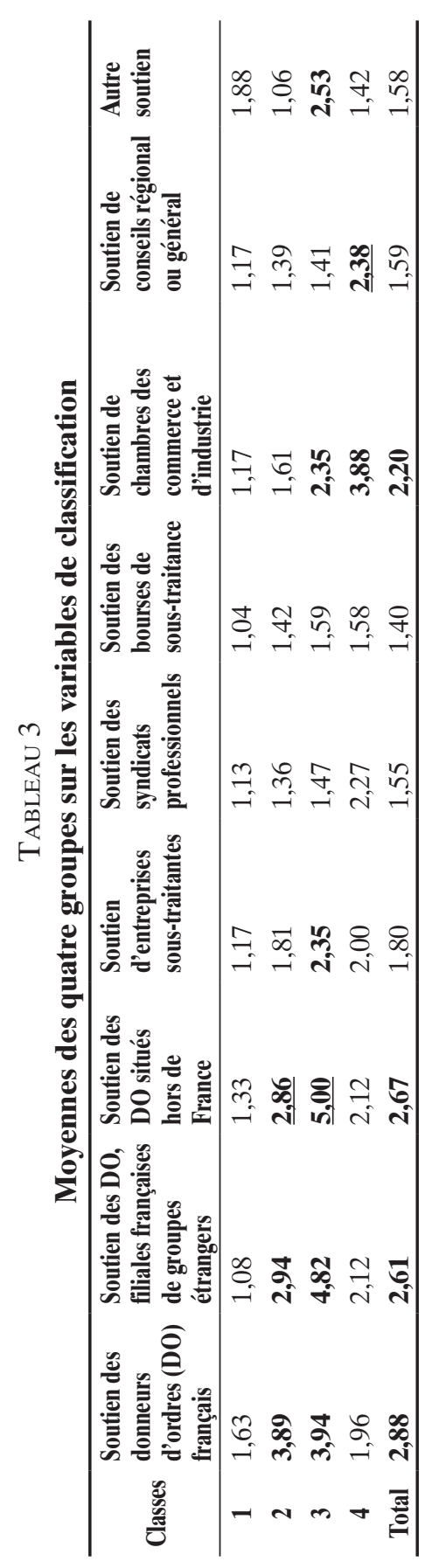

Revue internationale P.M.E., vol. 20, ns 3-4, 2007

() 2008 - Presses de l'Université du Québec

Édifice Le Delta I, 2875, boul. Laurier, bureau 450, Québec, Québec G1V 2M2 • Tél.: (418) 657-4399 - www.puq.ca 
3) Les plus soutenus (16,5\%), qui connaissent un développement international fortement soutenu, à la fois par leurs donneurs d'ordres (domestiques et étrangers) et les organismes institutionnels.

4) Les solliciteurs du réseau institutionnel (25\%), qui obtiennent des scores moyens concernant le soutien des chambres de commerce et d'industrie, du conseil régional et/ou général supérieurs aux scores respectifs atteints par les trois autres groupes. En revanche, la fréquence du soutien des donneurs d'ordres reste nettement inférieure à la moyenne de l'ensemble des observations.

Grâce à l'étude quantitative, nous observons que 90 \% des entreprises étudiées ont bénéficié, à des degrés divers, d'un soutien à l'international de leurs donneurs d'ordres et, dans une moindre mesure, des organismes institutionnels.

\subsection{Deux logiques d'internationalisation dominantes: induite réactive et entrepreneuriale}

L'analyse qualitative menée auprès des dirigeants de ces firmes exemplaires révèle l'importance de trois facteurs :l'origine interne ou externe du stimulus ayant déclenché la décision d'exporter, l'intensité du soutien à l'exportation émanant du réseau d'affaires de l'entreprise et l'engagement des ressources en interne au cours des phases lors du déploiement de l'activité internationale. Les quatre classes peuvent être rassemblées en deux grandes logiques d'internationalisation (voir figure 1).

\section{Figure 1}

La ventilation des modalités d'internationalisation en deux grandes logiques comportementales

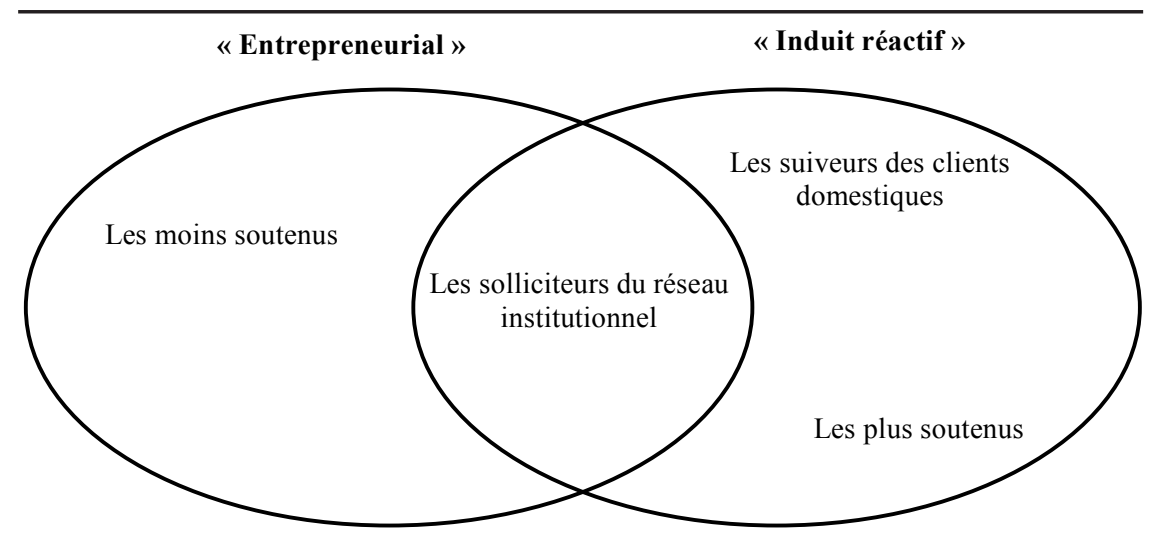

Revue internationale P.M.E., vol. 20, $\mathrm{n}^{\text {os }} 3-4,2007$ 
La première logique dite «induite réactive» domine dans cinq cas (Plastitrempage et Mécasoudure de la classe 2 «Les suiveurs de donneurs d'ordres domestiques», Labométa et Forgélec de la classe 3 «Les plus soutenus » et Laserdécoup de la classe 4 «Les solliciteurs du réseau institutionnel»). Burgelman (1983) rappelle que les «réactifs » se révèlent incapables de faire face aux mutations de leur environnement avec efficacité; ils s'adaptent lorsqu'ils sont soumis à de fortes pressions émanant de leur environnement. Dans les cas observés, le processus est initié par un stimulus externe, tel que le réseau de donneurs d'ordres ou la commande spontanée (tableau 4). Les dirigeants des PMI sous-traitantes ont tendance à réitérer les mêmes opérations. Dans ces conditions, le déroulement du processus peut être régulier (Labométa et Forgélec), jusqu'au moment où la survenue d'événements imprévus, essentiellement la perte de clients étrangers, introduit une rupture dans son déroulement (trois clients états-uniens pour Plastitrempage, quatre/cinq clients allemands pour Mécasoudure et un gros client belge pour Laserdécoup). L'entreprise entame alors la phase suivante du déroulement de son processus d'internationalisation grâce à d'autres solutions identifiées et retenues. 


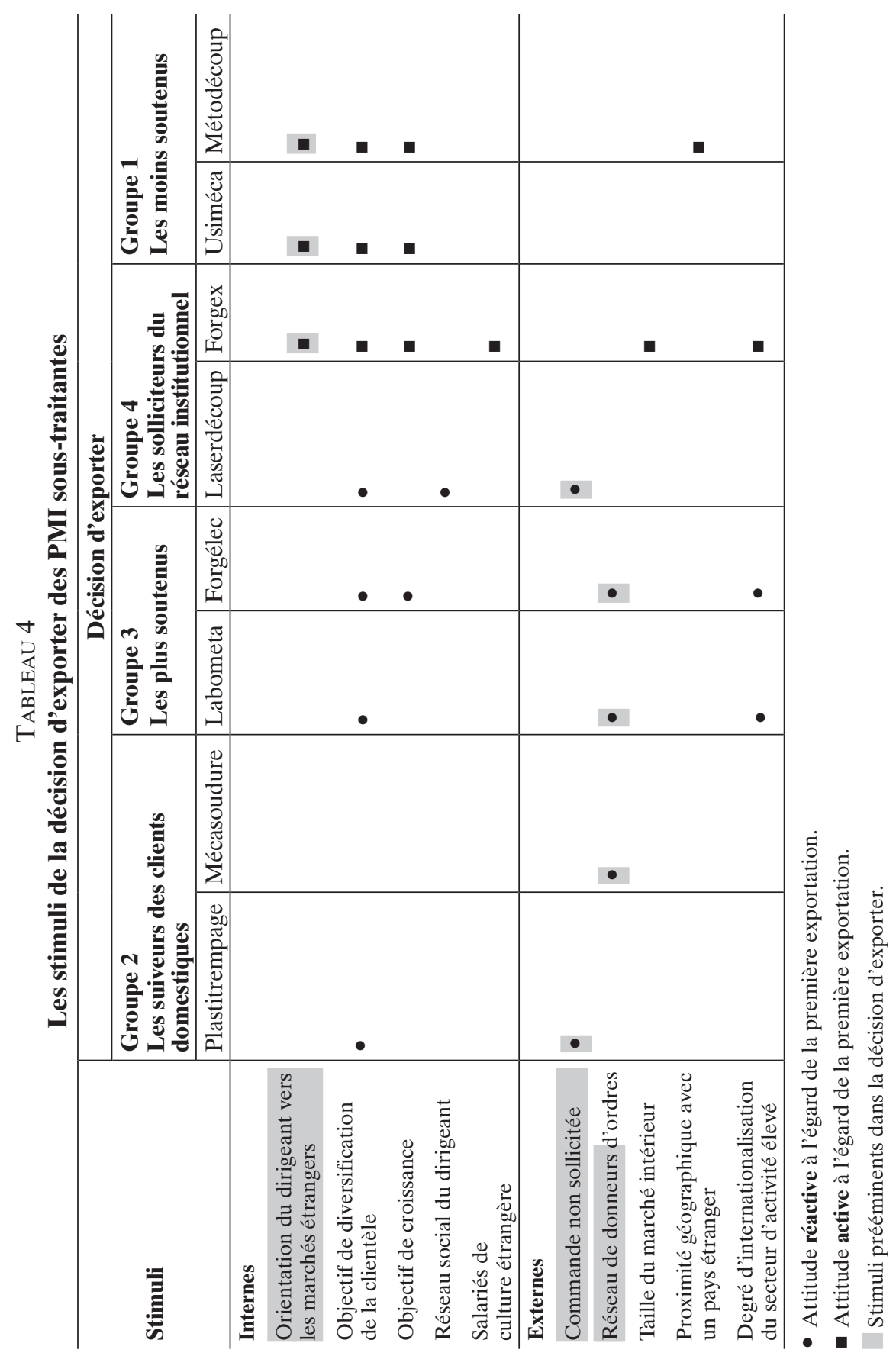

Revue internationale P.M.E., vol. 20, $\mathrm{n}^{\text {os }} 3-4,2007$ 
Il s'agit d'engager des ressources supplémentaires et/ou de solliciter le soutien du réseau institutionnel. Ces nouvelles solutions sont plus ou moins efficaces selon les sites: succès pour Plastitrempage et demi-échec pour Laserdécoup et Mécasoudure. Finalement, cette logique accélère le démarrage de l'exportation en levant les freins psychologiques. En revanche, elle se traduit par une attitude passive des dirigeants qui n'affichent pas la volonté de développer leur activité à l'international. Ne favorisant pas la mobilisation des ressources en interne, l'assistanat du réseau d'affaires inhibe l'apprentissage à l'exportation. Les taux d'exportation atteints sont alors nettement plus faibles que dans les situations d'internationalisation «entrepreneuriale» (voir le tableau 2 plus haut). Dans ce cas de figure, les dirigeants évitent le risque. La croissance de leur affaire est réactive, en ce sens qu'elle n'est acceptée que pour autant qu'elle ne remet pas en cause sa pérennité. Ils sont en attente d'occasions d'affaires mais ne les recherchent pas activement et perçoivent l'exportation comme une activité subsidiaire de leur activité domestique.

La seconde logique d'internationalisation, qualifiée «d'entrepreneuriale», caractérise Usiméca, Métodécoup (deux entreprises de la classe 1 «Les moins soutenus») et Forgex (une entreprise de la classe 4 «Les solliciteurs du réseau institutionnel»). Cette logique, au cœur de notre problématique, illustre l'existence d'un lien entre modalités d'internationalisation et développement, par les PMI sous-traitantes, d'un comportement entrepreneurial. Elle est approfondie ci-après.

\section{Focalisation sur les cas entrepreneuriaux}

Une meilleure compréhension du comportement entrepreneurial des PMI observées est autorisée par l'identification de traits partagés et distinctifs. Elle est précédée d'une présentation concise des trois cas entrepreneuriaux (tableau 5) s'appuyant sur le discours du dirigeant exprimé lors de l'entretien semi-directif, ses réponses préalables au questionnaire et des documents sectoriels issus de la littérature professionnelle. 


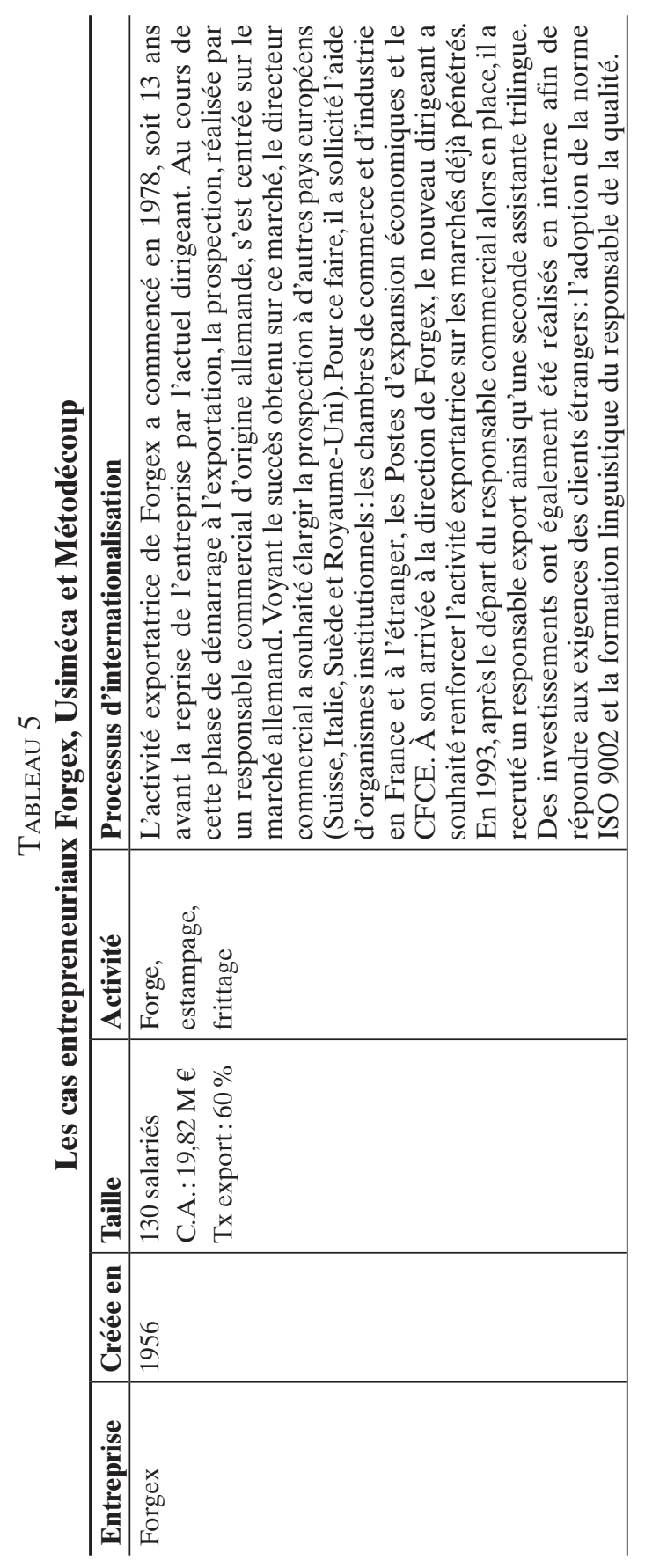

Revue internationale P.M.E., vol. 20, nºs 3-4, 2007 


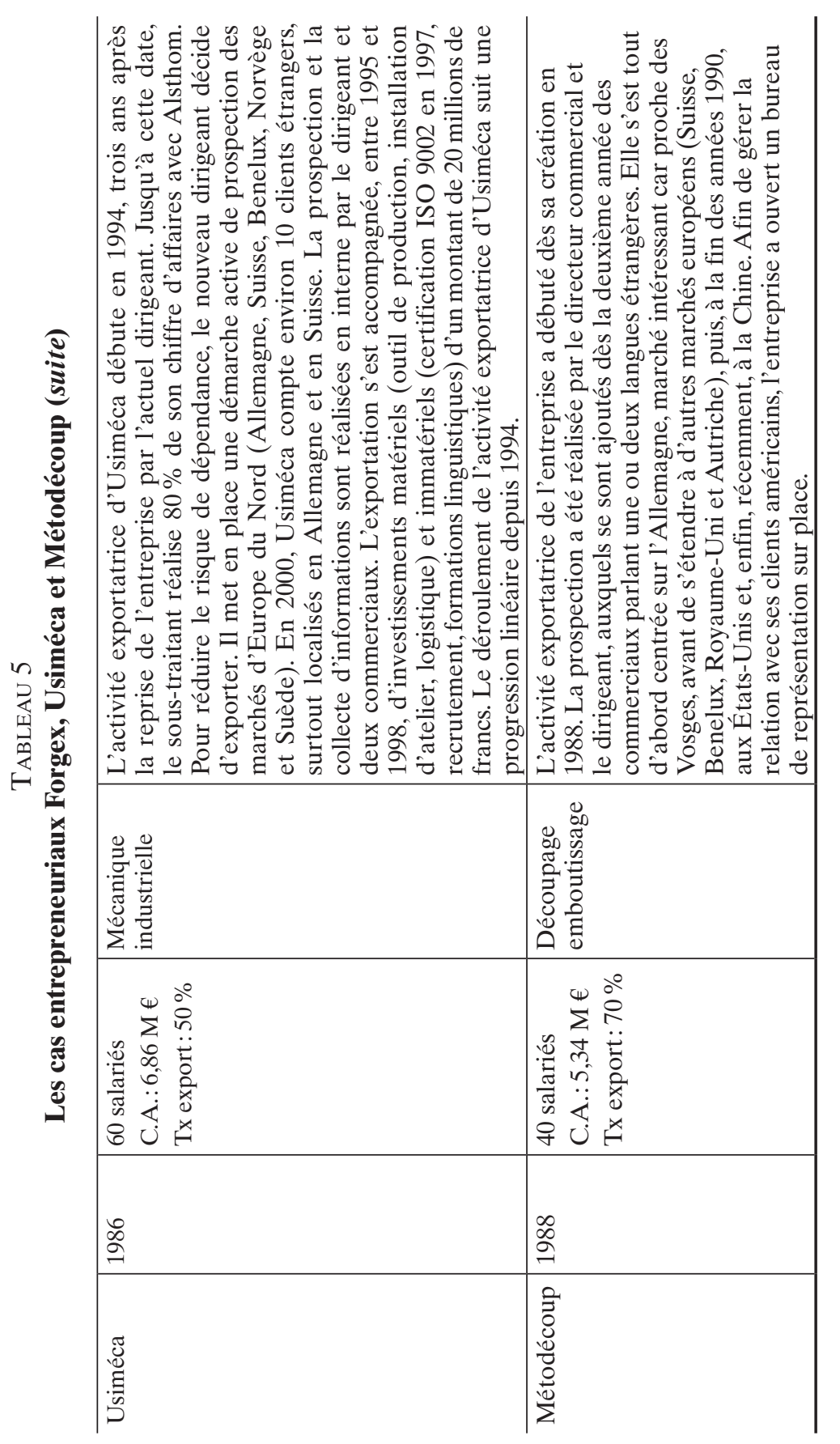

Revue internationale P.M.E., vol. 20, $\mathrm{n}^{\text {os }}$ 3-4, 2007 


\subsection{Les traits partagés}

L'analyse approfondie du processus d'internationalisation des trois situations «entrepreneuriales» fait apparaître de fortes similitudes qui les discriminent des cinq autres cas dominés par la logique «induite réactive». Trois des quatre dimensions entrepreneuriales citées par la littérature sont, à la différence des autres cas, effectivement constatées: une dynamique de création et de développement, la recherche d'occasions d'affaires et la création de valeur. L'innovation en revanche n'est pas une variable discriminante observée au regard de l'ensemble des cas étudiés.

Pour les sous-traitants entrepreneuriaux, la dynamique de création et de développement est patente. Le déclencheur de la décision d'exporter est interne. Il s'agit de l'orientation du dirigeant vers les marchés étrangers qui se traduit par une recherche délibérée et systématique de débouchés à l'exportation (tableau 4). Le démarrage du courant d'affaires à l'exportation se fait beaucoup plus graduellement. Il débute nécessairement par une commande test qui débouche, généralement au bout de cinq ans, sur une relation commerciale stable. À la différence des autres cas au processus d'internationalisation erratique (les réactifs dans §3.2), les trois cas entrepreneuriaux suivent, sous l'effet de l'expérience internationale (décrit par le modèle Uppsala), un processus d'expansion internationale progressif des marchés les plus proches aux marchés les plus éloignés psychologiquement. Possédant une véritable expérience à l'exportation sur les marchés européens, ces trois entreprises exportent (depuis la fin des années 1990 pour Métodécoup qui a implanté un bureau de représentation aux États-Unis puis en Chine) ou envisagent d'exporter vers des marchés plus éloignés géographiquement et culturellement (le Moyen-Orient ou l'Asie pour Usiméca et les États-Unis pour Forgex). Le processus d'internationalisation de ces PMI traduit un apprentissage à l'exportation, la sélection des marchés étrangers n'étant pas imposée par la localisation du réseau (filiales, maison mère, fournisseurs) des donneurs d'ordres intervenus dans l'internationalisation de la PMI sous-traitante.

Peu ou pas du tout soutenues par leur réseau d'affaires, ces entreprises peuvent exporter grâce à la mobilisation de ressources internes, source de création de valeur à l'international. L'ensemble des investissements (recrutement de personnes bilingues, organisation de formation linguistique, participation à des salons internationaux, réalisation de documents commerciaux en langues étrangères) s'articule dans le temps pour converger vers l'intensification de l'activité internationale. Cette dynamique d'évolution aboutit à des niveaux de performance à l'exportation, mesurée par le taux d'exportation, particulièrement élevés (tableau 5). 
Pour les dirigeants de ces entités, l'exportation constitue un enjeu stratégique. Chacun a su créer des opportunités à l'international:

Cas Forgex: «Il s'agissait de trouver d'autres marchés et d'autres clients [...] Pour nous, l'export nous a permis de maintenir notre volume d'activité dans des marchés très irréguliers ou fluctuants. Quand un marché est en baisse, il s'agit de se positionner sur ceux qui restent stables ou qui progressent.»

Cas Usiméca: «Dans notre stratégie de diversification, il y avait aussi une stratégie très nette qui était celle du développement de l'export. Aujourd'hui, on travaille en direct à plus de $60 \%$ à l'export et d'ailleurs si on ajoute les projets que l'on fait pour nos clients, je pense que l'on est à $95 \%$ au global [...] C'est là que je situe les marchés potentiels.»

Cas Métodécoup : «Métodécoup travaille à l'export depuis l'année de sa création. Comme je l'ai dit, c'était un objectif premier [...] L'exportation reste donc notre priorité.»

Quand le dirigeant n'aborde la question de la distance culturelle que pour les marchés non européens et, plus particulièrement, états-uniens ou asiatiques, la relation de sous-traitance internationale avec des donneurs d'ordres européens n'est pas différenciée d'une relation de sous-traitance domestique. C'est le cas des trois PMI sous-traitantes qui, sur ce dernier point, se distinguent des cinq autres cas étudiés.

Cas Forgex : «Nos clients sont tous Européens. Les mentalités sont donc très proches et ainsi les différences observables ne posent pas de problème particulier [...] Pour les marchés plus éloignés comme les États-Unis, au-delà de la distance géographique, se pose pour nous un problème juridique et législatif important, notamment la nécessité de trouver un avocat avant d'entreprendre toute démarche commerciale.»

Cas Usiméca : «Je dirais qu'actuellement, vu les marchés sur lesquels on travaille, on ne rencontre pas de difficultés, ni de correspondance, ni de langue, ni d'échange de monnaies [...] Maintenant peut-être que le discours serait modéré si on travaillait plus en direct sur notamment les pays asiatiques parce que, là, il y a un choc de cultures qui est plus important, mais ça c'est qu'une habitude à prendre.»

Cas Métodécoup: «Et puis, dans ces pays (États-Unis et Chine), on a un bureau sur place, qui a juste une fonction relationnelle. Quand vous êtes sur de tels marchés, vous êtes obligé d'avoir quelqu'un sur place, vous ne pouvez pas faire constamment des allers-retours. Pour pénétrer des marchés étrangers, il faut être rapide et réactif. Je ne vois pas d'autres différences au niveau de la relation commerciale entre la France et l'étranger.» 


\subsection{Les traits différenciateurs}

Au-delà des traits communs, il existe des traits différenciateurs qui font émerger des profils entrepreneuriaux quelque peu distincts.

Des différences sont repérées dans l'histoire de l'entreprise et de son dirigeant. Deux dirigeants sur trois (Forgex et Usimeca) sont des repreneurs, l'autre est un créateur. Le dirigeant de Forgex n'a pas initié l'exportation contrairement aux deux autres mais il a renforcé cette activité qui existait déjà lors de la reprise. Les raisons qui sous-tendent l'initiation de l'activité exportatrice dans les deux autres cas sont distinctes. Pour le dirigeant d'Usimeca, il s'agissait de limiter la dépendance de l'entreprise à un seul grand donneur d'ordres domestique: «Nous avons voulu la diversification, parce que jusqu'en 1994, la société était à $80 \%$ pour le compte d'une seule des divisions Alsthom et nous avons imaginé que c'était très dangereux à terme.» Pour le dirigeant de Métodécoup, l'exportation s'est imposée comme une priorité dès la création de l'entreprise : «Il fallait aller chercher le travail là où il se trouvait. Nous sommes dans une région pour ainsi dire désertique. Nous avons donc pris l'habitude d'aller chercher nos clients en Europe, puis de plus en plus loin, au fur et à mesure que nos compétences à l'export s'affermissaient.»

Un deuxième trait différenciateur provient de la nature de l'activité exercée par le sous-traitant, les trois entreprises appartenant à des secteurs d'activité aux règles du jeu distinctes. Forgex, dont l'activité est la forge à chaud, fournit des petites pièces (entre 0,5 et 1,5 kilogramme) en moyennes et grandes séries à de grands industriels principalement des secteurs automobile et poids lourd. Le rapport de force lui est défavorable et les relations avec ses donneurs d'ordres restent distantes et s'inscrivent dans le court terme: «Nos clients nous imposent des conditions strictement liées à des considérations de rentabilité. Si elles ne sont plus satisfaisantes, le client change de sous-traitant. Même avec nos plus vieux clients allemands, la notion de partenariat à long terme est un leurre.» L'activité d'Usimeca est regroupée autour de trois pôles: la mécano-soudure, l'usinage et le montage d'ensembles et de sous-ensembles mécaniques de grande dimension (entre 5 et 80 tonnes), à haute valeur ajoutée qui sont préparés soit sur le site du sous-traitant, soit chez le client, que ce dernier soit localisé en France ou à l'étranger. Les cycles de fabrication ont une durée de 4 à 8 mois suivant les projets. Usiméca fabrique des turbines vapeur pour ses clients traditionnels appartenant au secteur de l'énergie hydraulique, thermique et nucléaire. Pour ses clients sidérurgistes européens, elle fabrique des pièces de rechange et assure l'entretien des installations. En raison de la nature de son activité, Usiméca, à l'inverse de Forgex, entretient des relations partenariales avec ces

Revue internationale P.M.E., vol. 20, $\mathrm{n}^{\text {os }} 3-4,2007$ 
donneurs d'ordres : «On est associé totalement à la réflexion en amont avec un objectif qui est commun. C'est vraiment des relations franches et cordiales [...] Beaucoup ont fait l'expérience de délocaliser des fabrications dans des pays exotiques ou à faible coût de main-d'œuvre, mais ils ont beaucoup de soucis et en reviennent beaucoup.»

La modalité d'internationalisation mise en œuvre est un autre critère de différenciation. Ainsi, les trois cas entrepreneuriaux observés en profondeur diffèrent selon la classe d'appartenance. Forgex, contrairement aux deux autres cas (représentatifs de la classe des moins soutenus), appartient à la classe des solliciteurs du réseau institutionnel. Le dirigeant de cette entreprise a une très bonne connaissance des aides mises à disposition par le réseau institutionnel et, par conséquent, le sollicite à bon escient. Usiméca appartient à la classe des moins soutenus. L'absence de sollicitation des réseaux de donneurs d'ordres et institutionnel est motivée par la nature de son activité et la culture de son dirigeant. «On s'est pris par la main, on n'est surtout pas passé par un quelconque dispositif d'agents intermédiaires. Je savais que ça n'apporte rien [...] En plus, dans le cadre d'Usiméca, on vend du savoir-faire [...] Et puis, si vous voulez, moi, [...] je suis d'origine lorraine. Donc, nous on vit depuis tout petit aux frontières de trois pays. On est habitué à parler non pas seulement en français mais aussi en allemand et en anglais.» En revanche, pour Métodécoup, le choix d'une internationalisation autonome est ici lié, de l'avis de son dirigeant, au manque de pertinence des services proposés «Nous pensons qu'il n'y a aucun retour sur investissement. Nous avons déjà sollicité l'aide d'organismes, comme la chambre de commerce dans le passé, mais sans résultat.»

Enfin, des différences apparaissent dans le style de management du dirigeant qui impulse et organise. Le dirigeant de Forgex, le plus qualifié des trois $(\mathrm{bac}+5)$, est le seul à avoir délégué l'activité export à un autre responsable, sans doute en raison de la taille de son entité. Par ailleurs, il sollicite le réseau institutionnel sans s'y impliquer, refusant systématiquement de participer aux journées d'informations dont il ne perçoit pas l'intérêt immédiat pour son entreprise. Les relations qu'il entretient avec les donneurs d'ordres sont également distantes, essentiellement du fait de l'activité de son entreprise. Enfin, les relations avec d'autres sous-traitants sont uniquement de type concurrentiel. Le style de management du dirigeant d'Usiméca est, quant à lui, de nature «gagnant-gagnant». Les relations avec les donneurs d'ordres sont partenariales, qualifiées de cordiales et transparentes: "Nos clients nous rendent très fréquemment visite à Saint-Étienne et même ils viennent de n'importe quel coin de l'Europe [...] pour travailler sur des évolutions ou des nouveaux projets.» En 1998, le dirigeant d'Usiméca a envisagé la cotraitance. Il est toujours ouvert à la démarche mais sa recherche 
d'un partenaire sous-traitant n'a pas encore abouti. Enfin, le dirigeant de Métodécoup, le moins qualifié des trois $(\mathrm{bac}+2)$, est autonome dans sa façon de gérer. Il s'implique totalement dans le processus d'internationalisation en mobilisant son intuition et son savoir-faire empirique. Son style de management est de nature «donnant-donnant». Les relations avec les donneurs d'ordres sont strictement professionnelles. Elles ne durent que dans la mesure où sous-traitant et donneur d'ordres en tirent un intérêt: «Quand la relation est bonne, le client a intérêt à ce qu'elle perdure. Mais si demain il y a un problème, le client n'aura aucun état d'âme pour changer de soustraitant.» Par ailleurs, Métodécoup se sert de ses clients comme références: «Il fallait prouver à nos éventuels donneurs d'ordres qu'ils pouvaient nous faire confiance [...] Ils ont été satisfaits [...] Et puis, on s'est servi de ces clients-là pour nous référencer vers d'autres donneurs d'ordres.» Il s'investit également dans la relation dans la mesure où elle s'avère indispensable. À l'étranger, les échanges techniques et commerciaux sont gérés depuis la France et le dirigeant n'hésite pas à rendre visite à ses clients sur place. Métodécoup a par ailleurs essayé à deux reprises de s'allier à d'autres PMI sous-traitantes, sans succès «parce que chacun dirige son entreprise comme il l'entend, avec ses propres états d'âme. Aujourd'hui [...] nous n'assumons que notre savoir-faire, pas celui des autres».

Au regard de ces traits différenciateurs, il est alors possible de qualifier chacun des trois profils entrepreneuriaux observés:

- Forgex: un entrepreneuriat «distant et professionnel»;

- Usiméca: un entrepreneuriat «partenarial»;

- Métodécoup: un entrepreneuriat «autonome et impliqué».

Il est désormais intéressant de comparer ces profils aux résultats d'autres études consacrées au comportement entrepreneurial de PME qui s'internationalisent. Le nombre de travaux investissant ce champ est à ce jour restreint. Le travail d'Andersson (2000), notamment, autorise une comparaison intéressante. La typologie qu'il propose présente en effet trois profils de PMI entrepreneuriales qui, sans être sous-traitantes, s'internationalisent: l'entrepreneur technicien, l'entrepreneur marketing et l'entrepreneur structurel. L'entrepreneur technicien s'internationalise par le réseau technologique international. Nos cas ne privilégient pas la dimension technologique dans leur stratégie de développement et ne peuvent donc s'inscrire dans ce premier type. L'entrepreneur marketing décèle un besoin au sein de marchés étrangers et saisit cette opportunité pour développer son portefeuille de clientèle. Nos trois cas se situent dans ce deuxième type (trait partagé : la recherche d'opportunités). L'entrepreneur structurel se trouve dans des industries matures au 
marché obligatoirement international. L'internationalisation n'est donc pas un choix mais une condition d'existence. Sa stratégie de croissance interne par l'acquisition d'organisations nouvelles est source d'opportunités. Aucun de nos cas, du fait de leur qualité de sous-traitant, n'appartient à ce dernier type. Ainsi, nos résultats enrichissent un des types de la typologie d'Andersson en distinguant trois profils au sein du type entrepreneur marketing. Ce constat est incontestablement lié à la nature des PMI étudiées: sous-traitantes pour notre étude.

\section{Conclusion}

L'objectif initial de cet article était de proposer une lecture entrepreneuriale de l'internationalisation d'entreprises sous-traitantes de taille restreinte. Cette lecture s'est révélée intéressante à plusieurs titres. D'abord, elle infirme la thèse de l'association systématique d'une dimension stratégique et entrepreneuriale des PMI sous-traitantes qui ont choisi de se développer à l'international. Parmi les huit cas exemplaires issus de l'analyse typologique et étudiés en profondeur, seules les entreprises Forgex, Usiméca et Métodécoup manifestent un comportement entrepreneurial, les autres adoptent une attitude de type induit et réactif, subissant les pressions de leur environnement, de leurs donneurs d'ordres notamment. Notre lecture discrimine par ailleurs la modalité d'internationalisation sous-tendue par un comportement entrepreneurial: l'internationalisation de type autonome, non soutenue par les donneurs d'ordres. Lorsqu'elles ne sont pas ou peu entrepreneuriales, les PMI sous-traitantes observées s'avèrent être les plus soutenues à l'international par le réseau des donneurs d'ordres mais aussi les moins performantes à l'exportation. Le réseau accélère dans un premier temps leur démarrage à l'exportation sans autoriser, à la différence des cas entrepreneuriaux, un apprentissage progressif et incrémental. Elle offre enfin une première piste d'exploration de la diversité de profils entrepreneuriaux. Leur nature plus ou moins partenariale procède, dans les cas étudiés, du style de management impulsé par le dirigeant et des contraintes sectorielles existantes.

Ces résultats, issus du contexte singulier de la sous-traitance, mériteraient d'être comparés à ceux de recherches exclusivement consacrées à des PME exportatrices non sous-traitantes. La composante culturelle française et l'organisation du réseau institutionnel en France caractérisent également notre recherche. Une autre comparaison intéressante consisterait à étudier une population de PME issues d'autres pays européens tels que l'Allemagne ou les pays scandinaves dotés d'une forte culture à l'international. Les différents profils entrepreneuriaux détectés appellent d'autres travaux complémentaires sous forme d'études de cas approfondies. À ce titre, il conviendrait 
de s'interroger sur l'adoption de comportements distincts selon que l'entreprise se situe sur le marché domestique ou à l'étranger. À titre d'exemple : une PMI sous-traitante pourrait-elle adopter à l'international un comportement induit réactif, cependant que sur son marché domestique elle démontre un comportement entrepreneurial?

\section{Bibliographie}

Ageron, B. (2001), «La construction d'une capacité de réponse d'une PMI à l'international: une mise à l'épreuve des faits du modèle de Bilkey et Tesar », Revue internationale $P M E$, vol. 14, $\mathrm{n}^{\circ}$ 2, p. 9-39.

Altersohn, C. (1992), De la sous-traitance au partenariat industriel, Paris, L'Harmattan.

Andersen, P.H., P. Blenker et P.R. Christensen (1995), «Internationalization of subcontractors: in search of theoretical framework», Communication au RENT $I X$, Research on Entrepreneurships, Piacenga, $24 \mathrm{p}$.

Andersson, S. (2000), «The internationalization of the firm from an entrepreneurial perspective», International Studies of Management \& Organization, vol. 30, $\mathrm{n}^{\circ} 1$, p. 63-92.

BARbat, V. (1996), «Fédérer ses fournisseurs: la stratégie de Ford Blanquefort», Revue internationale de l'Achat, vol. 16, $\mathrm{n}^{\circ}$ 1, p. 11-14.

BARbat, V. (2004), Modalités et processus d'internationalisation des PMI sous-traitantes françaises: une étude multi-méthodes, Thèse de doctorat ès sciences de gestion, Université Montesquieu Bordeaux IV.

BAUdRY, B. (1991), «Une analyse économique des contrats du partenariat industriel: l'apport de l'économie des coûts de transaction », Revue d'économie industrielle, $\mathrm{n}^{\circ} 56, \mathrm{p} .46-57$.

BAUDRY, B. (1999), «Qualité des produits et coordination dans la relation de soustraitance: une analyse économique de la procédure de certification industrielle», Revue internationale PME, vol. 12, nº 4, p. 57-75.

BELL, J. (1995), «The internationalization of small computer software firms : a further challenge to stage theories », European Journal of Marketing, vol. 29, $\mathrm{n}^{\circ} 8$, p. $60-75$.

Bilkey, W. et G. Tesar (1977), «The export behavior of smaller sized Wisconsin manufacturing firms », Journal of International Business Studies, printemps-été, p. 93-98.

Bloodgood, J., H.J. SAPIENZA et J.G. Almeida (1996), «The internationalization of new high-potential U.S. ventures: antecedents and outcomes», Entrepreneurship: Theory and Practice, vol. 20, p. 61-76.

Bruyat, C. (1993), Création d'entreprise: contributions épistémologiques et modélisation, Thèse de doctorat en sciences de gestion, Université Pierre-Mendès-France de Grenoble.

Revue internationale P.M.E., vol. 20, $\mathrm{n}^{\text {os }} 3-4,2007$ 
Burgelman, R.A. (1983), «Corporate entrepreneurship and strategic management: insights from a process study», Management Science, vol. 29, $\mathrm{n}^{\circ} 2$, p. 1349-1364.

Bygrave, W. et C. Hofer (1991), «Theorizing about entrepreneurship», Entrepreneurship Theory and Practice, hiver, p. 13-22.

Commission des Communautés européennes (1992), Étude sur le poids économique et l'évolution de la sous-traitance dans la Communauté, Rapport de recherche pour la Direction générale XXIII, novembre.

Courault, B. (2005), «PME et industrialisation: que sont devenues les PME du "miracle choletais" (1945-2004)?», Centre d'études de l'emploi, n 53, décembre, $42 \mathrm{p}$.

Coviello, N.E. et A. McAuley (1999), «Internationalization and the smaller firm: a review of contemporary empirical research», Management International Review, vol. 39, no 3, p. 223-256.

Coviello, N.E. et H.J. MunRo (1995), «Growing the entrepreneurial firm: networking for international market development», European Journal of Marketing, vol.29, p. 49-61.

Coviello, N.E. et H.J. Munro (1997), «Network relationships and the internationalization process of small software firms », International Business Review, vol. 6, p. 361-386.

DALGiC, T., D. Li et L. Li (2004), «Internationalization process of small and mediumsized enterprises: towards a hybrid model of experiential learning and planning», Management International Review, vol. 44, n 1, p. 93-116.

Dichtl, E., M. Liebold, H. Köglmayr et S. Müller (1984), «Export decision of small and medium-sized firms: a review», Management International Review, vol. 24, p. 49-61.

FEnNETEAU, H. (1990), «Mise en concurrence des fournisseurs ou partenariat?», Revue internationale PME, vol. 3, $\mathrm{n}^{\circ}$ 2, p. 167-192.

Flechter, D. et S. Vyakarnam (1999), «Enacting globalisation and mapping growth in small entrepreneurial firms », Entrepreneurship Research Conference, Caroline du Sud, 11-15 mai, Babson College/Kauffman.

GARTNER, W. (1993), «Words lead to deeds: towards an organizational emergence vocabulary», Journal of Business Venturing, vol. 8, n 3, p. 231-239.

Harveston, P.D., B.L. Kedia et P.S. Davis (2002), «Internationalization of born global and gradual globalizing firms: the impact of the manager», Advances in Competitive Research, vol. 8, n 1, p. 92-99.

HolmLund, M. et S. Kоск (1998), «Relationships and the internationalisation of Finnish small medium-sized companies », International Small Business Journal, vol. $16, \mathrm{n}^{\circ} 4, \mathrm{p} .46-63$.

Hovi, N. (1994), «Outcomes of interfirm co-operation case: internationalising subcontractors », IMP Conference.

Revue internationale P.M.E., vol. 20, nos 3-4, 2007 
Huberman, A.M. et M.B. Miles (2003), Analyse des données qualitatives: recueil de nouvelles méthodes, Bruxelles, De Boeck Université, $2^{\mathrm{e}}$ édition en français.

JAFFE, E.D. et H. PASTERNAK (1994), «An attitudinal model to determine the export intention of non exporting small manufacturers», International Marketing Review, vol. 11, n 3 , p. 17-32.

JohAnsson, J. et L.G. MATtsson (1988), «Internationalisation in industrial systems: a network approach », dans N. Hood et J.-E. Vahlne (dir.), Strategies in Global Competition, Londres, Croom Helm, p. 287-314.

JOHANSSON, J. et J.E. VAHLNE (1977), «The internationalization process of the firm: a model of knowledge development and increasing foreign market commitments », Journal of International Business Studies, vol. 8, n 1, p. 23-32.

JoHANsSon, J. et J.E.VAHLNe (2003), «Business relationship learning and commitment in the internationalization process », Journal of International Entrepreneurship, vol. 1, p. 83-101.

JohANSSOn, J. et F. Wiedersheim-PAUL (1975), «The internationalization of the firm: four Swedish cases», Journal of International Business Studies, octobre, p. 305-322.

Julien, P.-A. et M. Marchesnay (1989), La petite entreprise, Paris, Vuibert.

LAVAL, F. (1994), La gestion des ressources humaines des entreprises fournisseurs - partenaires: l'impact de la politique d'achat d'un grand groupe industriel, Thèse pour le doctorat nouveau régime en sciences de gestion, Université de Montpellier II.

Lloyd-Reason, L. et T. Mughan (2002), «Internationalization within SME's: the key role of owner-manager», Journal of Small Business and Enterprise Development, vol. 9, n 2, p. 120-130.

McDougall, P.P. et B.M. Oviatt (2000), «International entrepreneurship : the intersection of two research paths », Academy of Management Journal, vol. 43, n 5 , p. 902-906.

McDougall, P.P. et B.M. Oviatt (2005), «Defining international entrepreneurship and modeling the speed of internationalization ", Entrepreneurship Theory and Practice, septembre, p. 537-553.

McDougall, P.P., S. Shane et B.M. Oviatt (1994), «Explaining the formation of internationalisation new ventures: the limits of theory from international business research », Journal of Business Venturing, vol. 6, nº 6, p. 469-487.

Ministère de L'ÉCONOMIE, DES FinANCES ET DE L'INDUSTRIE (2000), La sous-traitance en chiffres: métaux, plastiques, électronique, Service des études et des statistiques industrielles - SESSI.

Ministère DE L'ÉCONOMIE, DES FINANCES ET DE L'INDUSTRIE (2003), La sous-traitance en chiffres, Service des études et des statistiques industrielles - SESSI, numéro hors série des synthèses chiffrées de «production industrielle».

Nummela, N. (1997), «The significance of business network in small business internationalisation», IMP Conference.

Revue internationale P.M.E., vol. 20, $\mathrm{n}^{\text {os }} 3-4,2007$

(C) 2008 - Presses de l'Université du Québec

Édifice Le Delta I, 2875, boul. Laurier, bureau 450, Québec, Québec G1V 2M2 • Tél.: (418) 657-4399 - www.puq.ca

Tiré de: Revue internationale P.M.E., vol. $20, \mathrm{n}^{\circ \mathrm{s}} 3-4$, sous la direction de Louis Raymond $\bullet$ PME2003N Tous droits de reproduction, de traduction et d'adaptation réservés 
REID, S. (1981), «The decision maker and export entry and expansion», Journal of International Business Studies, automne, p. 101-112.

Rispal, M. (1993), Les modes de création et de fonctionnement d'accords de coopération (AC) transnationaux entre dirigeants de PME-PMI européens: une analyse qualitative inductive, Thèse de doctorat ès sciences de gestion, Université de Bordeaux I.

RonsDadt, R. (1984), Entrepreneurship, Dover, Lord Publishing.

SHANE, S. et S. VenKatARAmAn (2000), «The promise of entrepreneurship as a field of research», Academy of Management Review, vol. 25, n 1, p. 217-226.

Shrader, R.C., B.M. Oviatt et P.P. McDougall (2000), «How new ventures exploit trade-offs among international risk factors: lessons for the accelerated internationalization of the 21st century », Academy of Management Journal, vol. 43, p. $1227-1248$.

STEVENSON, H. et J. JARILlO (1990), «A paradigm of entrepreneurship: entrepreneurial management», Strategic Management Journal, vol. 11, p. 17-27.

Stewart, W.H., W.H.WATSON, J.C. CARLAnd et J.W. CARLAND (1999), «A proclivity for entrepreneurship: a comparison of entrepreneurs, small business owners and corporate managers », Journal of Business Venturing, vol. 14, n 2, p. 189-214.

Thornton, P. (1999), «The sociology of entrepreneurship», Annual Review of Sociology, vol. 25, p. 19-26.

L'USINE NouvelLe (1996), Sous-traitance, le défi de l'international, dossier réalisé sous la direction de D. Coué, no 2572, novembre, p. 52-81.

L'USINE NouvelLe (2003), Dossier sous-traitance, $\mathrm{n}^{\circ}$ 2893, novembre, p. 64-90.

Verstraete, T. et A. Fayolle (2005), «Quatre paradigmes pour cerner le domaine de recherche en entrepreneuriat», Revue de l'Entrepreneuriat, vol. 4, $\mathrm{n}^{\circ} 1$, p. 33-52.

Wiedersheim-Paul, F., H.C. Olson et L.S. Welch (1978), «Pre-export activity: the first step in internationalisation», Journal of International Studies, printemps-été, p. 47-56.

WolfF, J.A. et T.L. PetT (2000), «Internationalization of small firms : an examination of export competitive patterns, firm size and export performance», Journal of Small Business Management, vol. 38, nº 2, p. 34-47. 